\title{
Avaliação da concordância diagnóstica entre métodos não invasivos e endoscopia na investigação de infertilidade
}

\author{
Evaluation of the diagnostic agreement between non invasive methods and endoscopy in \\ infertility investigation \\ Luciana de Souza Borges ${ }^{1}$, Júlio César Rosa e Silva ${ }^{2}$, Ana Carolina Japur de Sá Rosa e Silva², Flávia Maciel \\ Aguiar $^{1}$, Omero Benedicto Poli Neto ${ }^{3}$, Francisco José Candido dos Reis ${ }^{4}$, Antonio Alberto Nogueira ${ }^{4}$
}

\section{RESUMO}

Objetivo: avaliar a concordância entre os métodos não invasivos - queixa de dor pélvica, ultra-sonografia transvaginal (US-TV) e histerossalpingografia (HSG) e a endoscopia ginecológica no diagnóstico de fatores tubo-peritoneais responsáveis por infertilidade conjugal. Métodos: foi realizado estudo do tipo corte transversal incluindo 149 pacientes inférteis submetidas à avaliação clínica, ultra-sonografia transvaginal, histerossonografia, histeroscopia e laparoscopia. $\mathrm{Na}$ avaliação de dor pélvica foram consideradas anormais a queixa de dor pélvica tipo dispareunia, dismenorréia ou dor acíclica e dor à mobilização do colo uterino e palpação de anexos. $\mathrm{O}$ exame ultra-sonográfico foi considerado alterado com os achados de alterações morfológicas anexiais ou uterinas (hidrossalpinge, miomas ou malformações uterinas). A histerossalpingografia foi considerada anormal na presença de alteração anatômica tubária e obstrução unilateral ou bilateral. Avaliou-se a concordância diagnóstica individual entre os diversos métodos não invasivos e a endoscopia por meio da análise kappa. Resultados: a concordância entre dor pélvica, US-TV e HSG e avaliação endoscópica foi, respectivamente, de 46,3\% ( $\kappa=0,092$; IC 95\%: $-0,043$ a 0,228$), 24 \%$ ( $\kappa=-0,052$; IC 95\%: $-0,148$ a 0,043) e 46\% ( $\kappa=0,092$; IC 95\%: -0,043 a 0,228). Quando se considerou pelo menos um método não invasivo positivo, a concordância com a avaliação endoscópica foi de 63\% ( $\kappa=-0,014$; IC 95\%: -0,227 a 0,199). A sensibilidade e especificidade em predizer achados na endoscopia foram de 39,5 e 80\% na presença de dor pélvica, de 14,5 e 72\% na presença de alteração na US-TV, de 39,5 e $80 \%$ na presença de alteração na HSG e de 70,2 e $28 \%$ na presença de ao menos uma alteração na avaliação não invasiva. Conclusão: há fraca concordância diagnóstica entre os diversos métodos não invasivos e a endoscopia na investigação de infertilidade conjugal secundária a fatores tubo-peritoneais.

PALAVRAS-CHAVE: Laparoscopia; Histerossalpingografia; Ultrasonografia; Dor pélvica; Infertilidade feminina

\section{ABSTRACT}

Purpose: to evaluate the agreement between noninvasive methods - pelvic pain, transvaginal ultrasound and hysterosalpingography - and the gynecologic endoscopy approach for the diagnosis of tuboperitoneal factors responsible for conjugal infertility. Methods: this is a cross-sectional study including 149 infertile patients who were submitted to clinical evaluation, transvaginal ultrasound, hysterosalpingography, hysteroscopy, and laparoscopy. In the evaluation of pelvic pain, the following complaints were considered to be abnormal: pelvic pain of the dyspareunia type, dysmenorrhea or acyclic pain, and pain upon mobilization of the cervix and palpation of the adnexa. Ultrasonographic examination was considered to be altered when adnexal or uterine morphological changes (hydrosalpinx, myomas or uterine malformations)

Serviço de Endoscopia Ginecológica do Departamento de Ginecologia e Obstetrícia da Faculdade de Medicina de Ribeirão Preto, Universidade de São Paulo - USP - Ribeirão Preto (SP) - Brasil.

1 Pós-graduando do Departamento de Ginecologia e Obstetrícia do Hospital das Clincas da Faculdade de Medicina de Ribeirão Preto, Universidade de São Paulo - USP - Ribeirão Preto (SP) - Brasil.

2 Médico Assistente do Hospital das Clinicas da Faculdade de Medicina de Ribeirão Preto, Universidade de São Paulo - USP - Ribeirão Preto (SP) - Brasil.

3 Docente do Departamento de Cirurgia e Anatomia da Faculdade de Medicina de Ribeirão Preto, Universidade de São Paulo - USP - Ribeirão Preto (SP) - Brasil.

4 Docente do Departamento de Ginecologia e Obstetrícia da Faculdade de Medicina de Ribeirão Preto, Universidade de São Paulo - USP - Ribeirão Preto (SP) - Brasil.

Correspondência: Antonio Alberto Nogueira

Departamento de Ginecologia e Obstetrícia - Av. Bandeirantes, 3900 - Campus da USP - 14049-900 - Ribeirão Preto-SP - Telefone: (16) 602-2804 e-mail: aanoguei@fmrp.usp.br 
were detected. Hysterosalpingography was considered to be abnormal in the presence of anatomical tubal changes and unilateral or bilateral obstruction. The agreement between noninvasive methods and endoscopy was evaluated by kappa statistics. Results: the agreements between pelvic pain, transvaginal ultrasound, and hysterosalpingography and the endoscopic approach were $46.3 \%(\kappa=0.092$; CI 95\%: -0.043 to 0.228$), 24 \%$ ( $\kappa=-0.052$; CI $95 \%$ : -0.148 to 0.043$)$, and $46 \%$ $(\kappa=0.092$; CI 95\%: -0.043 to 0.228$)$, respectively. When at least one alteration detected by noninvasive methods was considered, the agreement with endoscopic approach was $63 \%(\kappa=-0.014$; CI $95 \%$ : -0.227 to 0.199$)$. Sensitivity and specificity in predicting alterations on endoscopic approach were 39.5 and $80 \%$ in the presence of pelvic pain, 14.5 and $72 \%$ in the presence of alteration on transvaginal ultrasound, 39.5 and $80 \%$ in the presence of alteration on hysterosalpingography, and 70.2 and $28 \%$ in the presence of at least one alteration by noninvasive methods. Conclusion: there is a poor diagnostic agreement between the several noninvasive methods and endoscopy in the investigation of conjugal infertility secondary to tuboperitoneal factors.

KEYWORDS: Laparoscopy; Hysterosalpingography; Ultrassonography; Pelvic pain; Infertility, female

\section{Introdução}

A infertilidade conjugal afeta de 10 a $15 \%$ dos casais em idade reprodutiva ${ }^{1}$, sendo as doenças do trato genital feminino responsáveis por 50 a $60 \%$ dos casos, ao passo que 40 a $50 \%$ estão relacionados a fatores masculinos. Dentre as causas femininas destacam-se os fatores tubo-peritoneais, associados à doença inflamatória pélvica, endometriose, cirurgias anteriores, abortos sépticos, apendicite com peritonite e prenhez ectópica, que apresentam prevalência variável na dependência do perfil epidemiológico da população estudada ${ }^{2-5}$. Diversos métodos propedêuticos são propostos para sua investigação, dentre os quais se destacam a histerossalpingografia (HSG) e a laparoscopia (LPS) (Quadro 1). Destes, a LPS é o método mais sensivel e específico na investigação dos fatores tubo-peritoneais, mas está associada aos riscos inerentes a um procedimento cirúrgico invasivo.

Quadro 1 - Achados positivos segundo o tipo de variável estudada.

\begin{tabular}{|ll|}
\hline Variável & Achado positivo \\
\hline Avaliação clínica & História de doença inflamatória \\
& pélvica, algia pélvica e cirurgias \\
& pélvicas anteriores.Exame ginecológi- \\
& co doloroso. \\
Ultra-sonografia & Hidrossalpinge (unilateral ou bilateral), \\
transvaginal & alteração uterina (miomas e \\
& malformações) e ovariana (cistos e \\
& ovários policísticos). \\
Histerossalpingografia & $\begin{array}{l}\text { Alteração anatômica tubária e obstru- } \\
\text { ção unilateral ou bilateral. }\end{array}$ \\
Laparoscopia diagnóstica & $\begin{array}{l}\text { Doença tubária intrínseca } \\
\text { (tortuosidade, dilatação, aglutinação } \\
\text { de fímbrias e obstrução tubária), ade- } \\
\text { rências pélvicas, endometriose, } \\
\text { miomas e malformações uterinas e } \\
\text { ovários policísticos. }\end{array}$ \\
\hline
\end{tabular}

Rev Bras Ginecol Obstet. 2005;27(7):401-6
Os critérios para a indicação de LPS previamente a procedimentos de reprodução assistida não são consensuais na literatura atual ${ }^{6,7}$. Quando a HSG é anormal ou existem fatores de risco e sintomas associados sugestivos de comprometimento tubo-peritoneal, a LPS é altamente recomendada, pois, além da maior eficácia diagnóstica, pode ser também método terapêutico para diversas doenças como endometriose e aderências ${ }^{8}$. Entretanto, quando a HSG é normal e a probabilidade de doença adicional é baixa, a laparoscopia pode ser adiada ou mesmo substituída por métodos alternativos não cirúrgicos na investigação da infertilidade, como histerossalpingossonografia contrastada e a ressonância nuclear magnética que, conforme proposto, se normais, poderiam dispensar a investigação cirúrgica de rotina 9 .

Aqueles que preconizam a não-realização de procedimentos endoscópicos em pacientes assintomáticas e com exames menos invasivos como a HSG normais argumentam que nesta situação a presença de alterações pélvicas clinicamente relevantes é muito baixa, e que com as técnicas de reprodução assistida disponíveis o impacto do tratamento cirúrgico de eventuais achados de endometriose mínima ou leve tornouse insignificante ${ }^{10}$. Por outro lado, aqueles mais liberais com a aplicação dos procedimentos endoscópicos na investigação da infertilidade feminina argumentam que os procedimentos de reprodução assistida não são de acesso fácil e na maioria das vezes não são cobertos pelos planos de saúde, considerando que pequenas melhoras nas taxas de gravidez em função de tratamento cirúrgico de endometriose mínima e leve ou de aderências tubo-ovarianas devem ser consideradas importantes ${ }^{11}$. 
Este trabalho tem como objetivo avaliar a concordância entre os métodos não invasivos queixa de dor pélvica, ultra-sonografia transvaginal (US-TV) e HSG - e a endoscopia no diagnóstico de condições que levam à infertilidade por comprometimento tubo-peritoneal.

Métodos

Foi realizado estudo observacional do tipo corte transversal, incluindo 149 pacientes inférteis referidas para o serviço terciário de Reprodução Humana do Departamento de Ginecologia e Obstetrícia da Faculdade de Medicina de Ribeirão Preto da Universidade de São Paulo, no período de junho de 1999 a dezembro de 2000. Durante este periodo, todas as pacientes inférteis foram submetidas à LPS e histeroscopia previamente a quaisquer procedimentos de reprodução assistida. O trabalho foi aprovado pelo Comitê de Ética em Pesquisa do Hospital das Clínicas da Faculdade de Medicina de Ribeirão Preto da Universidade de São Paulo.

Definiu-se infertilidade conjugal como a incapacidade de concepção após um ano de vida sexual ativa e regular, sem uso de método contraceptivo.

A média de idade das pacientes neste estudo foi de 29,9 anos, variando entre 16 e 41 anos, sendo que 36 pacientes $(24,1 \%)$ apresentavam 35 anos ou mais. Em relação ao tipo de infertilidade, 99 pacientes $(66,4 \%)$ apresentavam infertilidade primária e $50(33,6 \%)$ infertilidade secundária. Quanto ao tempo de infertilidade, 73 pacientes (49\%) tinham entre 1 e 4 anos e 76 (51\%) mais de 4 anos. Foi observado ao menos um tipo de achado intra-operatório que justificasse a infertilidade em $72,5 \%$ das pacientes, ou seja, 108 das 149 pacientes incluídas neste estudo.

$\mathrm{Na}$ avaliação de dor pélvica foram consideradas anormais a queixa de dor pélvica tipo dispareunia, dismenorréia ou dor acíclica e dor à mobilização do colo uterino e palpação de anexos. O exame ultra-sonografico foi considerado alterado com o achado de imagem anexial sugestiva de hidrossalpinge (unilateral ou bilateral) ou presença de alterações morfológicas uterinas (mioma e malformações). A HSG foi considerada anormal na presença de alteração anatômica tubária e obstrução unilateral ou bilateral.

Todas as pacientes foram submetidas à avaliação endoscópica em nosso serviço e os procedi- mentos documentados de forma sistematizada em formulários próprios para relato de laparoscopia e histeroscopia. A histeroscopia foi considerada alterada na presença de lesões intracavitárias como pólipos, miomas ou sinéquias. Foram considerados anormais os achados laparoscópicos de alteração anatômica tubária como tortuosidade, dilatação, aglutinação de fimbrias ou obstrução tubária, presença de aderências pélvicas envolvendo as trompas ou ovários e presença de endometriose (Quadro 1).

O resultados dos métodos de investigação foram analisados de forma dicotomizada (normal ou anormal). Foi considerada concordância positiva quando os dois métodos comparados eram anormais e concordância negativa quando ambos eram normais.

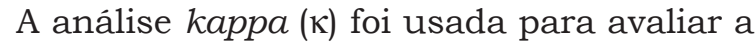
concordância individual entre os diversos métodos não invasivos de investigação de infertilidade e a endoscopia ginecológica. A interpretação foi dada conforme os valores obtidos: $\kappa<0,20$ : concordância fraca; $0,21 \leq \kappa<0,40$ : concordância razoável; $0,41 \leq \kappa<0,60$ : concordância moderada; $0,61 \leq \kappa<0,80$ : concordância boa; $0,81 \leq \kappa<1,00$ : concordância muito boa $^{12}$.

\section{Resultados}

A concordância entre dor pélvica e avaliação endoscópica foi de 46,3\%. A concordância foi positiva em 49 casos e negativa em 20 casos ( $\kappa=0,092$; IC 95\%: -0,043 a 0,228). Entre os casos discordantes a dor pélvica apresentou 75 falso-negativos e 5 falso-positivos. A sensibilidade da dor pélvica para predizer alteração endoscópica foi de $39,5 \%$ e a especificidade de $80 \%$ (Tabela 1 ).

Tabela 1- Achados anormais na laparoscopia.

\begin{tabular}{lcc}
\hline Doenças & $\mathbf{n}$ & $\%$ \\
\hline Endometriose mínima/leve & 46 & 58,2 \\
Endometriose moderada/grave & 15 & 18,9 \\
Aderências pélvicas & 28 & 35,4 \\
Doença intrínseca tubária & 11 & 13,9 \\
Miomas & 9 & 11,3 \\
Ovários policísticos & 15 & 18,9 \\
\hline
\end{tabular}


A US-TV mostrou concordância com a avaliação endoscópica em $24 \%$ dos casos, sendo a concordância positiva em 18 pacientes e a negativa em 18 casos $(\kappa=-0,052$; IC 95\%: $-0,148$ a 0,043). Entre os casos discordantes a US-TV apresentou sete falso-positivos e 106 falso-negativos. A sensibilidade da US-TV para predizer alteração endoscópica foi de $14,5 \%$ e a especificidade de $72 \%$.

A HSG mostrou concordância com a avaliação endoscópica em $46 \%$ dos casos, sendo a concordância positiva em 49 casos e negativa em 20 casos ( $\kappa=0,092$; IC 95\%: $-0,043$ a 0,228). Entre os casos discordantes a HSG apresentou 75 falsonegativos e cinco falso-positivos. A sensibilidade da HSG para predizer alteração endoscópica foi de $39,5 \%$ e a especificidade de $80 \%$.

Quando se considerou pelo menos um método não invasivo positivo, houve concordância com a avaliação endoscópica em $63 \%$ dos casos, sendo a concordância positiva em 87 casos e negativa em sete casos ( $\kappa=-0,014$; IC 95\%: -0,227 a $0,199)$. Entre os casos discordantes a combinação de métodos não invasivos apresentou 37 falso-negativos e 18 falso-positivos. A sensibilidade combinada dos métodos não invasivos para predizer alteração endoscópica foi de $70,2 \%$ e a especificidade de $28 \%$.

\section{Discussão}

Em nosso estudo verificamos fraca concordância entre os diagnósticos obtidos por meio dos diversos métodos não invasivos e a endoscopia na investigação de infertilidade conjugal. Neste contexto há necessidade de se fazerem diversas considerações.

A utilização do sintoma dor para predição da ocorrência de alterações pélvicas durante a avaliação endoscópica não foi eficaz, a sensibilidade é inferior a $40 \%$ e a sua ausência não pode por si ser considerada como critério para a não realização de investigação endoscópica. Com especificidade de $80 \%$, a presença de dor crônica, tanto referida como observada pelo exame clínico, pode ser considerada importante critério para a indicação de endoscopia na mulher infértil. A história de dismenorréia e dispareunia aumentam a probabilidade de se encontrar endometriose de 41 para 64 e $69 \%$, respectivamente. A presença de ambos os sintomas aumentam a probabilidade para $83 \%{ }^{13}$.

A ultra-sonografia, outro método valioso na investigação da mulher infértil, também apre- sentou fraca concordância com a avaliação endoscópica. Como esperado, a sensibilidade é muito fraca principalmente para detecção de alterações tubo-peritoneais, que constituíram a maioria das doenças encontradas em nossa casuística. O uso de contraste intracavitário uterino para visualização ultra-sonográfica, histerossalpingossonografia, tem sido sugerido para o diagnóstico alternativo de alterações da permeabilidade tubária, com relatos de elevada acurácia ${ }^{14}$. Em alguns estudos seu desempenho é semelhante à $\mathrm{HSG}^{15}$. A ultra-sonografia simples normal não pode, no entanto, ser considerada como critério para não se realizar endoscopia. Por outro lado, a boa especificidade coloca este método, quando anormal, como um reforço na indicação de procedimento invasivo.

A HSG tem sua aplicação difundida na investigação básica do casal infértil, com baixo custo e facilidade de execução, podendo ser realizada em unidades de atendimento secundário de saúde, ao passo que a endoscopia é mais invasiva e apresenta maior custo, além de exigir serviço terciário de atendimento médico. A HSG permite apenas a avaliação da permeabilidade tubária e da amplitude da cavidade uterina, ao passo que a endoscopia permite a avaliação completa da cavidade uterina e da anatomia pélvica, em especial das trompas. O procedimento endoscópico possibilita o diagnóstico de doenças peritoneais como a endometriose, e da cavidade uterina como pólipos; permite ainda o tratamento destas condições em um mesmo tempo ${ }^{16}$. Em nosso estudo, parte das pacientes com HSG normal apresentou comprometimento tubo-peritoneal identificado após avaliação endoscópica, sendo a endometriose e as aderências pélvicas envolvendo trompas ou ovários as alterações mais freqüentes. A freqüência de nossos achados é compativel com os descritos por outros autores ${ }^{13}$. Nesta situação, mesmo que a infertilidade não seja completamente justificada pela doença encontrada à endoscopia, a facilidade da abordagem cirúrgica, uma vez exposta a cavidade abdominal, justifica a terapêutica, cuidando-se para não produzir impacto negativo sobre o prognóstico reprodutivo da paciente, como em ressecções de tecido ovariano.

Encontramos sensibilidade de $39,5 \%$ e especificidade de $80 \%$ da HSG para predizer alteração endoscópica. Na literatura podem ser encontrados estudos com sensibilidade de $65 \%{ }^{17} \mathrm{e}$ estudos com taxas mínimas de falso-negativos, como $3,6 \%^{3}$. Por outro lado, a boa especificidade 
principalmente na detecção de obstrução tubárea é mais consensual, com valores em torno de $80 \%{ }^{17}$. O resultado de HSG normal não exclui a presença de distorções anatômicas das trompas, porém se a HSG sugerir obstrução tubária provavelmente ela será confirmada pela LPS. Dessa forma, a presença de obstrução tubária diagnosticada na HSG poderia dispensar a investigação cirúrgica e indicar um procedimento de reprodução assistida; em contrapartida, a presença de HSG normal não seria suficiente para o diagnóstico, permanecendo os métodos endoscópicos como opção mais sensivel, principalmente para a detecção de endometriose mínima e leve e de aderências tubo-ovarianas.

Outra possível causa para a discordância entre a HSG e endoscopia encontrada neste estudo pode ser a variabilidade na interpretação dos achados. Entre as principais causas de falso-negativo na HSG podem ser ressaltados a técnica inadequada, o tipo de contraste e a interpretação do exame. Tentativas como a associação de cultura para Chlamidia trachomatis a uma HSG normal podem reduzir a taxa de falso-negativos para doença tubária de 15 para $5 \%$, porém com importante elevação dos custos ${ }^{8}$.

A difusão das técnicas de reprodução assistida muitas vezes dispensa a necessidade do diagnóstico preciso para o sucesso do procedimento ${ }^{18}$; neste contexto é aceitável se prescindir da laparoscopia em pacientes sem queixa ou achado clínico de dor pélvica, ultra-sonografia e HSG normal. Considerando-se, no entanto, um aspecto mais amplo da abordagem do casal infértil, acreditamos que a LPS é recurso extremamente eficaz para o diagnóstico de fatores tubo-peritoneais, bem como para a sua abordagem terapêutica, alem de ter importância na tomada de decisão pelo casal sobre qual será seu próximo passo no tratamento e, portanto, deve ser cuidadosamente considerada antes de estabelecer o diagnóstico final da causa de infertilidade.

\section{Não há conflito de interesse.}

Rev Bras Ginecol Obstet. 2005;27(7):401-6

\section{Referências}

1. Mosher WD, Pratt WF. Fecundity and infertility in the United States: incidence and trends. Fertil Steril. 1991;56(2):192-3.

2. Henig I, Prough SG, Cheatwood M, DeLong E. Hysterosalpingography, laparoscopy and hysteroscopy in infertility. A comparative study. J Reprod Med. 1991;36(8):573-5.

3. Opsahl MS, Miller B, Klein TA. The predictive value of hysterosalpingography for tubal and peritoneal infertility factors. Fertil Steril. 1993;60(3):444-8.

4. Randolph JF Jr, Ying YK, Maier DB, Schmidt CL, Riddick DH. Comparison of real-time ultrasonography, hysterosalpingography, and laparoscopy/hysteroscopy in the evaluation of uterine abnormalities and tubal patency. Fertil Steril. 1986;46(5):828-32.

5. Jaffe SB, Jewelewicz R. The basic infertility investigation. Fertil Steril. 1991;56(4):599-613.

6. Lavy Y, Lev-Sagie A, Holtzer H, Revel A, Hurwitz A. Should laparoscopy be a mandatory component of the infertility evaluation in infertile women with normal hysterosalpingogram or suspected unilateral distal tubal pathology? Eur J Obstet Gynecol Reprod Biol. 2004;114(1):64-8.

7. Shokeir TA, Shalan HM, El-Shafei MM. Combined diagnostic approach of laparoscopy and hysteroscopy in the evaluation of female infertility: results of 612 patients. J Obstet Gynaecol Res. 2004;30(1):9-14.

8. Corson SL, Cheng A, Gutmann JN. Laparoscopy in the "normal" infertile patient: a question revisited. J Am Assoc Gynecol Laparosc. 2000;7(3):317-24.

9. Ayida G, Chamberlain P, Barlow D, Koninckx P, Golding S, Kennedy S. Is routine diagnostic laparoscopy for infertility still justified? A pilot study assessing the use of hysterosalpingo-contrast sonography and magnetic resonance imaging. Hum Reprod. 1997;12(7):1436-9.

10. Fatum M, Laufer N, Simon A. Investigation of the infertile couple: should diagnostic laparoscopy be performed after normal hysterosalpingography in treating infertility suspected to be of unknown origin? Hum Reprod. 2002;17(1):1-3.

11. Papaioannou S, Afnan M, Olufowobi O, Sharif K. Investigation of the infertile couple: laparoscopy after normal hysterosalpingography? Hum Reprod. 2002;17(7):1928-9.

12. Mackinnon A. A spreadsheet for the calculation of comprehensive statistics for the assessment of diagnostic tests and inter-rater agreement. Comput Biol Med. 2000;30(3):127-34. 
13.al-Badawi IA, Fluker MR, Bebbington MW. Diagnostic laparoscopy in infertile women with normal hysterosalpingograms. J Reprod Med. 1999;44(11):953-7.

14. Hauge K, Flo K, Riedhart M, Granberg S. Can ultrasound-based investigations replace laparoscopy and hysteroscopy in infertility? Eur J Obstet Gynecol Reprod Biol. 2000;92(1):167-70.

15. Exacoustos C, Zupi E, Carusotti C, Lanzi G, Marconi $\mathrm{D}$, Arduini D. Hysterosalpingo-contrast sonography compared with hysterosalpingography and laparoscopic dye pertubation to evaluate tubal patency. J Am Assoc Gynecol Laparosc. 2003;10(3):367-72.
16. Gordts S, Campo R, Puttemans P, Verhoeven H, Gianaroli L, Brosens $\mathrm{J}$, et al. Investigation of the infertile couple: a one-stop outpatient endoscopybased approach. Hum Reprod. 2002;17(7):1684-7.

17. Swart P, Mol BW, van der Veen F, van Beurden M, Redekop WK, Bossuyt PM. The accuracy of hysterosalpingography in the diagnosis of tubal pathology: a meta-analysis. Fertil Steril. 1995;64(3):486-91.

18. Balasch $\mathrm{J}$. Investigation of the infertile couple: investigation of the infertile couple in the era of assisted reproductive technology: a time for reappraisal. Hum Reprod. 2000;15(11):2251-7. 